\title{
A 29-Year-Old Male with Borderline Lepromatous Leprosy
}

\author{
Lubna Khondker ${ }^{1}$, Md. Shirajul Islam Khan ${ }^{2}$, Md. Anwar Husain ${ }^{3}$
}

\begin{abstract}
Leprosy (Hansen's disease) is a chronic granulomatous infectious disease that primarily affects the peripheral nerves, skin, upper respiratory tract mucosa, eyes and certain other tissues. It is diagnosable and curable if recognized early and treated adequately. A twenty nine-year-old male from Jessore, Bangladesh reported in Bangabandhu Sheikh Mujib Medical University, Dhaka, Bangladesh with the complaints of multiple erythematous, large, raised and circumscribed lesions with loss of sensations on different parts of the body, especially distal portions of all four limbs for last eight months. Subsequently he developed ulcers on the anesthetic fingers due to smoking and few ulcerative lesions on both feet. Skin examination revealed multiple erythematous, large nodular lesions on both sides of the cheek and forehead, multiple erythematous, indurated, large plaques with raised margin and central clearing on the trunk, waist and all four limbs, few satellite lesions around the large plaques on the trunk, few hypopigmented patches and plaques on buttock and lower limbs, multiple painless ulcers on dorsal surface of fingers of both hands, both lateral malleoluses and right sole. On examination of peripheral nerves, left great auricular nerve, both ulnar nerves and both common peroneal nerves were moderately enlarged and tender. Slit skin smear for AFB (modified Z-N stain) was done and revealed that there were large number of acid and alcohol-fast bacilli arranged in straight and curved parallel bundles with globular masses (cigar-bundle appearance), morphologically resembling Mycobacterium leprae. Skin biopsy for histopathological examination revealed extensive infiltration of macrophages in the dermis, separated from epidermis by narrow grenz zone, with destruction of skin adnexa. Few foci of poorly defined granuloma in dermis were also noted. The patient was managed with rifampicin, clofazimine, dapsone, prednisolone and omeprazole.
\end{abstract}

Key words: Leprosy, Borderline lepromatous leprosy, Granulomatous diseases

J Enam Med Col 2013; 3(2): 109-112

\section{Introduction}

Leprosy (Hansen's disease) is a spectrum disease characterized by a variety of abnormal immune response, that primarily affects the peripheral nerves, skin, upper respiratory tract mucosa, eyes and certain other tissues. It is diagnosable and curable if recognized early and treated adequately. ${ }^{1}$ The causative agent is Mycobacterium leprae which was first identified in 1873 by the Norwegian bacteriologist Gerhard Henrik Armauer Hansen. ${ }^{2}$ It occurs at all ages but more common in between 10-
20 and in 30-60 years. ${ }^{3}$ The ratio of male and female is $2: 1$ and common in India, Brazil, Indonesia, Myanmar, Madagascar, Nepal, Bangladesh, Thailand, Sub-Saharan Africa and Central America. The prevalence in Bangladesh is $0.29 / 10000 .^{4}$ Whatever may be the route of transmission. bacteremia occurs in all forms of leprosy and bacteria disseminate throughout the body via blood and lymphatics. ${ }^{5}$ M. leprae enter the target tissue, selectively schwann cells of peripheral nerves where

1. Assistant Professor, Department of Dermatology \& Venereology, Bangabandhu Sheikh Mujib Medical University, Dhaka

2. Graded Specialist in Dermatology, Combined Military Hospital, Dhaka Cantonment, Dhaka

3. Associate Professor, Department of Dermatology, Ibn Sina Medical College, Dhaka 
they multiply and are liberated from the infected schwann cells and then infect neighboring schwann cells and thus intraneural infection spreads. ${ }^{6}$ The infected nerve is then invaded by histocytes (macrophage) and lymphocytes with subsequent formation of granuloma leading to nerve damage resulting neurological manifestations. ${ }^{7}$ If the body's cell mediated immunity (CMI) is capable of anchoring the infection within the nerves without skin involvement, this will be limited to pure neural leprosy. But if bacilli or antigens escape from the nerve into surrounding tissue, skin lesions of tuberculoid leprosy (TT) develop at that site. Subsequently borderline leprosy and lepromatous leprosy may develop depending upon the immunity of the individual. ${ }^{8}$ Tuberculoid leprosy patient has strong T-cell and macrophage activation with release of type-1 cytokine (IL-2, IFN-Y and IL-12) which results in CMI response and localizes the infection. In lepromatous leprosy, release of type- 2 cytokine (IL-4, IL-5, IL-10) causes strong antibody response and concomitantly inhibits T-cell and macrophage resulting in progression of the infection. ${ }^{9}$ It depends on the integrity of the host's specific cell mediated immunity (CMI) response to the $M$ leprae and it may be genetically determined. ${ }^{10}$

\section{Case report}

A 29-year-old, male from Kathaltola, Jessore, Bangladesh reported in the department of Dermatology and Venereology, Bangabandhu Sheikh Mujib Medical University, Dhaka, Bangladesh on 9 March 2010 with the complaints of multiple erythematous, large, raised and circumscribed lesions on different parts of the body for one year and loss of sensation on different parts of the body with multiple ulcers on both hands and feet for 8 months. According to the patient's statement, he was reasonably well one year back. Since then he had developed multiple large, reddish, raised and circular lesions on different parts of his body. Initilally the lesions were smaller in size, then gradually it became larger and spread on forehead, both sides of cheek, back of the trunk, waist and all four limbs. He also complained that he developed loss of sensations on different parts of the body especially distal portions of all four limbs for last eight months.

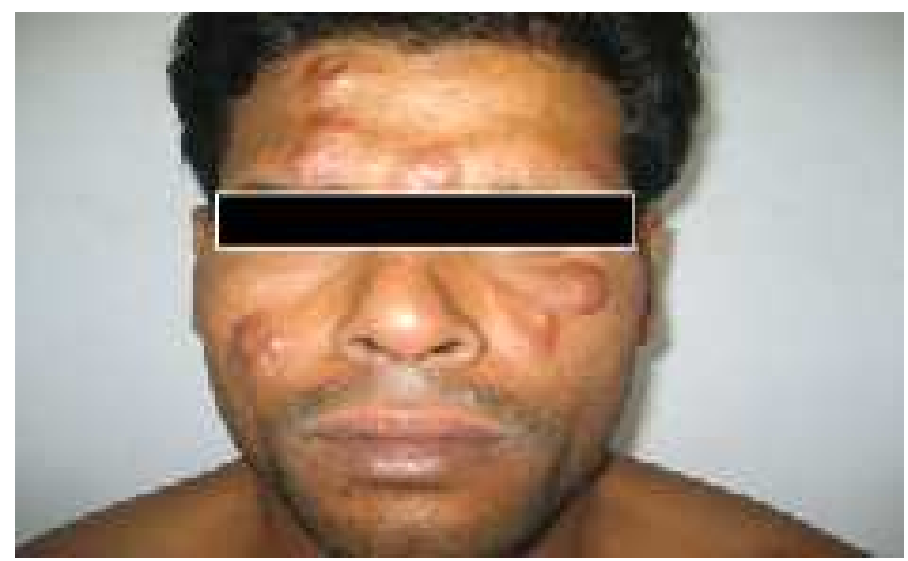

Fig 1: Multiple erythematous, large nodular lesions on both sides of the cheek and fore head

Subsequently he developed burn wounds on the anesthetic fingers due to smoking and few ulcerative lesions on both feet. Skin examination revealed multiple erythematous, large nodular lesions on both sides of the cheek and forehead, multiple erythematous, indurated, large plaques with raised margin and central clearing on the trunk, waist and limbs, few satellite lesions around the large plaques on the trunk, few hypopigmented patches and plaques on buttock and lower limbs, multiple painless ulcers on dorsal surface of fingers of both hands, both lateral malleoluses and right sole. On examination of peripheral nerves, left great auricular nerve was moderately enlarged and tender, both ulnar nerves were moderately enlarged and tender, both common peroneal nerves were moderately enlarged and tender; but no abnormality was detected in radial, median and posterior tibial nerves.

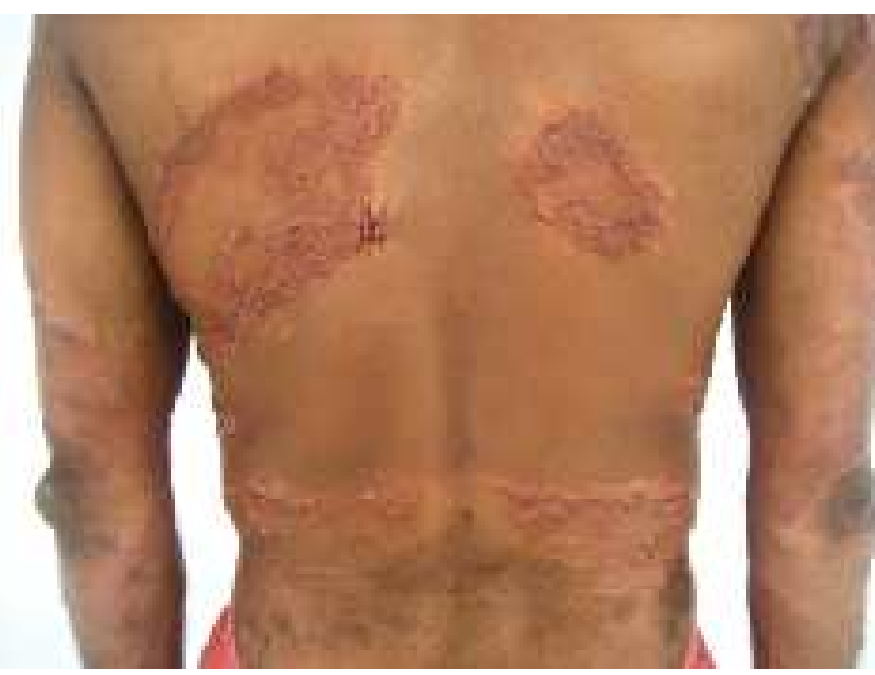

Fig 2: Multiple erythematous, indurated, large plaques with raised margin and central clearing on the trunk, waist and upper limbs 
Heat, cold, fine touch, pain and deep touch sensations were lost with glove-stocking anesthesia on all four limbs. Mucous membrane, hair and nail were intact. No abnormality was detected also in other systemic examinations.

\section{Laboratory investigations}

Mantoux test: negative, blood for glucose-6phosphate dehydrogenase: $272 \mathrm{mu} / 10^{9}$ erythrocytes, VDRL and TPHA: nonreactive, skin scraping for fungal microscopic examination showed no hyphe and culture showed no fungal growth. ICT result for Kala-Azar was negative. All hematological and biochemical tests were within normal limit. Slit skin smear for AFB (modified Z-N stain): Stained films revealed that there were large number of acid and alcohol-fast bacilli arranged in straight and curved parallel bundles with globular masses (cigar-bundle appearance), morphologically resembling Mycobacterium leprae.

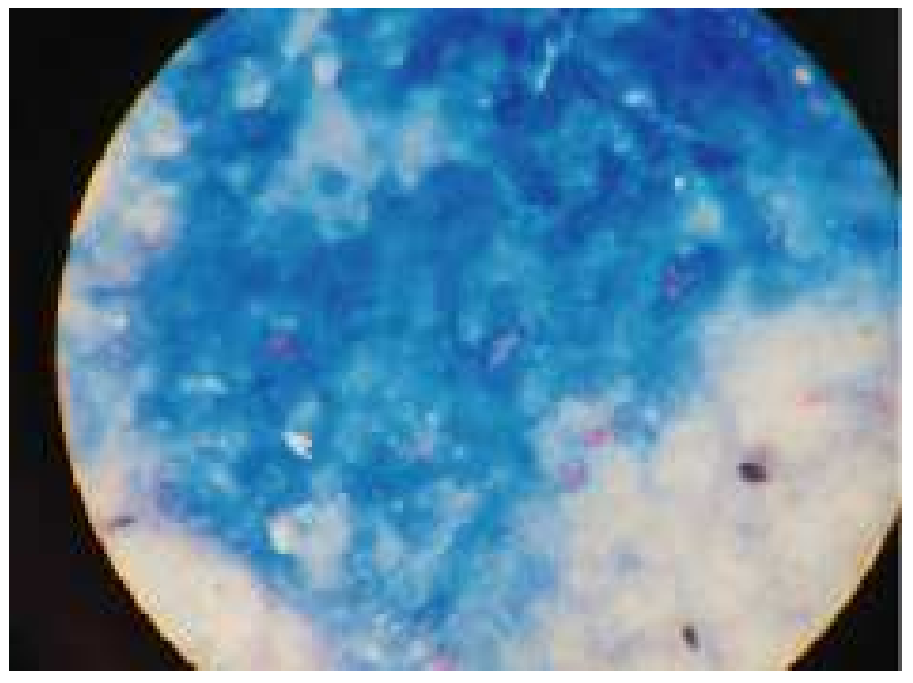

Fig 3: Slit skin smear for AFB (modified Z-N stain)

Skin biopsy for histopathological examination and Fite-Faraco stain: Section from skin revealed extensive infiltration of macrophage in the dermis, separated from epidermis by narrow Grenz zone, with destruction of skin adnexa. Few foci of poorly defined granuloma in dermis is also noted. Large number of lepra bacilli were seen in Wade-Fite stained sections.

The various differential diagnoses considered were PKDL, tinea corporis, lupus vulgaris, sarcoidosis and mycosis fungoides. However, based on clinical features, histopathological and microscopic examinations, borderline lepromatous leprosy was regarded as the most likely diagnosis.

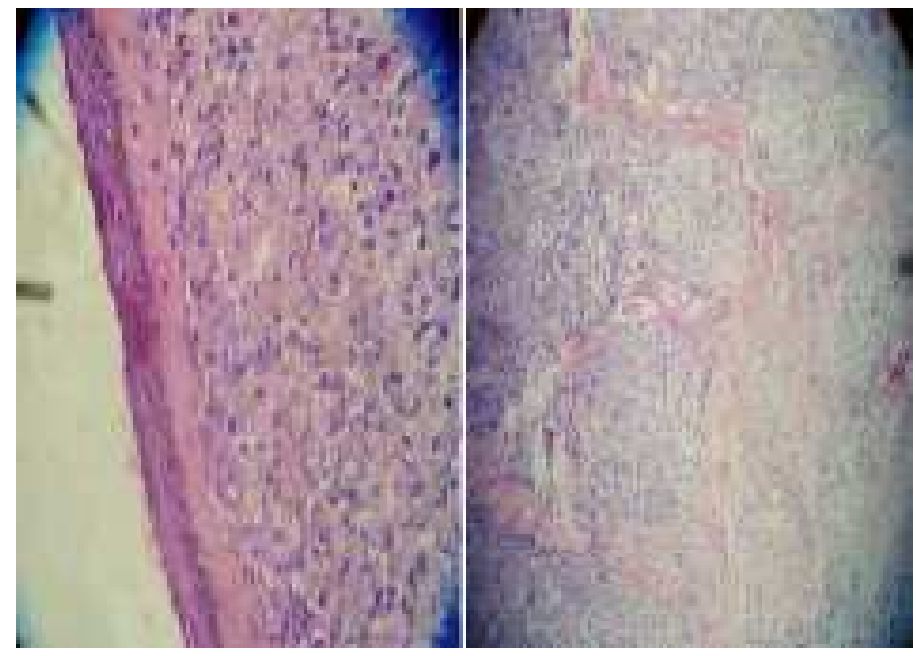

Fig 4: Findings of histopathological examination and Fite-Faraco stain

The patient was managed with rifampicin $600 \mathrm{mg}$ once in a month, clofazimine $300 \mathrm{mg}$ once in a month and $50 \mathrm{mg}$ daily, dapsone $100 \mathrm{mg}$ daily, prednisolone $40 \mathrm{mg}$ daily and omeprazole $40 \mathrm{mg}$ daily. Treatment was continued for one year.

\section{Discussion}

In borderline lepromatous leprosy, the lesions are symmetrical, numerous (too many to count), and may include macules, papules, plaques and nodules. ${ }^{2}$ This patient's lesions were symmetrical and morphologically similar which support the criteria of borderline lepromatous leprosy. Nerve involvement appears later, nerves are enlarged, tender or both, and it is important to note that involvement is symmetrical. ${ }^{2}$ This patient's nerve involvement fulfill these criteria. Sensation and sweating over individual lesions are normal. ${ }^{2}$ This patient's sensation and sweating over different types of lesions were also normal.

The findings of this case are consistent with typical presentation of borderline lepromatous leprosy. Histopathology of skin showed poorly defined granuloma in dermis with extensive infiltration of macrophages, which fit with the histopathological finding of borderline lepromatous leprosy. Complete clearance of nodular lesions, plaques, clean-looking ulcers after treatment with antileprosy regime is strongly suggestive of lepromatous origin. 


\section{References}

1. Rea TH, Modlin RL. Leprosy. In: Wolff K, Goldsmith LA, Katz SI, Gilchrest BA, Paller AS, Leffel DJ (eds). Fitzpatrick's dermatology in general medicine. $7^{\text {th }}$ edn. New York: McGraw Hill Companies, 2008: 1786-1796.

2. Hansen's disease. In: James WD, Berger TG, Elston DMS (eds). Andrews' diseases of the skin: clinical dermatology. $10^{\text {th }}$ edn. USA: Saunders Elsevier, 2006: 343-352.

3. Jopling WH, McDougall AC. Handbook of leprosy. $4^{\text {th }}$ edn. Oxford: Heinemann Professional Publishing, 1988: $18-45$.

4. Hastings RC. Leprosy. $2^{\text {nd }}$ edn. New York: Churchil Living Stone, 1994: 64-92.
5. Wolverton SE. Comprehensive dermatologic drug therapy. Philadelphia: W. B. Saunders Company, 2001: 230-250.

6. National guidelines and technical manual on leprosy. $3^{\text {rd }}$ edn. World Health Organization, 2005.

7. Madhusudan M. Leprous neuritis: a diagnostic dilemma. Indian J Dermatol Venereol Leprol 2009; 65(2): 59-65.

8. Thapa KS. Relapse in leprosy. Indian J Dermatol Venereol Leprol 2009; 75(2): 126-135.

9. Rajjal A, Rajjal S, Bhandari S. Leprosy coinfection with kala-azar. International Journal of Dermatology 2009; 48(7); 740-742.

10. Zodpey SP. Protective effect of BCG vaccine in the prevention of leprosy. Indian $\mathrm{J}$ Dermatol Venereol Leprol 2007; 73(2): 86-93. 